\title{
Alcove walks and nearby cycles on affine flag manifolds
}

\section{Ulrich Görtz}

Received: 4 October 2006 / Accepted: 2 February 2007 /

Published online: 7 April 2007

(C) Springer Science+Business Media, LLC 2007

\begin{abstract}
Using Ram's theory of alcove walks we give a proof of the Bernstein presentation of the affine Hecke algebra. The method works also in the case of unequal parameters. We also discuss how these results help in studying sheaves of nearby cycles on affine flag manifolds.
\end{abstract}

\section{Introduction}

\subsection{Main result}

In a recent paper [14], Ram has introduced the notion of alcove walk and used it in order to describe the affine Hecke algebra associated to a root datum.

In these notes we will show that, with a little extra work, this concept yields a proof of the Bernstein presentation of the affine Hecke algebra $\mathcal{H}$. The method applies to the case of unequal parameters as well (see Sect. 3.4), and we obtain a proof which might be considered less technical than the one given by Lusztig [13].

The main new ingredient beyond Ram's paper is the following theorem which we will state here in the introduction in a special case without using the language of alcove walks. Let $W_{a}$ be the affine Weyl group associated with some root system, and let $s_{0}, \ldots, s_{r}$ denote the simple reflections, which generate $W_{a}$. Denote by a the base alcove in the "standard apartment".

Theorem 1.1.1 Let $w \in W_{a}$. For an expression

$$
w=s_{i_{1}} \cdots s_{i_{n}}
$$

U. Görtz (凶)

Mathematisches Institut, Beringstr. 1, 53115 Bonn, Germany

e-mail: ugoertz@math.uni-bonn.de 
of $w$ as a product in the generators (which does not have to be reduced), consider the element

$$
\Psi(w):=T_{s_{i_{1}}}^{\varepsilon_{1}} \cdots T_{s_{i_{n}}}^{\varepsilon_{n}}
$$

in the affine Hecke algebra, where the $\varepsilon_{v} \in\{ \pm 1\}$ are determined as follows. Let $\mathbf{b}$ be an alcove far out in the anti-dominant chamber ("far out" depends on w, and the result will then be independent of $\mathbf{b}$, see Sect. 2.3 for a precise definition). For each $v$, consider the alcove $\mathbf{c}_{v}:=s_{i_{1}} \cdots s_{i_{v-1}} \mathbf{a}$, and denote by $H_{v}$ the affine root hyperplane containing its face of type $i_{v}$. We set

$$
\varepsilon_{v}= \begin{cases}1 & \text { if } \mathbf{c}_{v} \text { is on the same side of } H_{v} \text { as } \mathbf{b} \\ -1 & \text { otherwise }\end{cases}
$$

Then the element $\Psi(w)$ is independent of the choice of expression (1.1.1).

This is a special case of Theorem 3.1.1 in the text. It is implicitly contained in Ram's paper, and I actually learned its statement from Ram before his paper was finished. As an anonymous referee pointed out to me, the theorem (as stated above) follows immediately from Schwer's results, cf. [16] Lemma 6.1 and the discussion following it; see also [15].

We use this theorem to infer, from Ram's arguments, a proof of the Bernstein presentation of the affine Hecke algebra, starting from the Iwahori-Matsumoto presentation. Ram takes the Bernstein presentation as the definition of the affine Hecke algebra.

As a corollary to Theorem 3.1.1, we get the existence of so-called minimal expressions (cf. the paper [11] by Haines and Pettet) for the elements $\Theta_{\lambda} \in \mathcal{H}$. These are related to certain nearby cycles sheaves on the affine flag variety. See Sect. 4.

\section{The alcove walk algebra}

\subsection{Notation}

In this section we will collect the relevant notation; for more details on these notions, see Humphreys' book [12] and the papers by Lusztig [13], Haines and Pettet [11] and Haines, Kottwitz, and Prasad [9], for instance. Note that Lusztig uses a setup which is dual to ours: he works with roots where we use coroots, and conversely.

Let $\left(X^{*}, X_{*}, R, R^{\vee}, \Delta\right)$ be a reduced and irreducible based root datum with $\Delta$ being the set of simple roots, and denote by $W$ its Weyl group, generated by the simple reflections $\left\{s_{\alpha} ; \alpha \in \Delta\right\}$. Denote by $\widetilde{W}:=X_{*} \rtimes W$ the extended affine Weyl group. For $\lambda \in X_{*}$, we denote by $\epsilon^{\lambda}$ the corresponding element in $\widetilde{W}$. Let $S_{a}=\left\{s_{\alpha} ; \alpha \in \Delta\right\} \cup\left\{s_{0}\right\}$, where $s_{0}=\epsilon^{\tilde{\alpha}^{\vee}} s_{\tilde{\alpha}}$ and where $\tilde{\alpha}$ is the unique highest root. The subgroup $W_{a} \subseteq \widetilde{W}$ generated by $S_{a}$ is the affine Weyl group of the root system associated with our root datum, and $\left(W_{a}, S_{a}\right)$ is a Coxeter system.

We define a length function $\ell: \widetilde{W} \longrightarrow \mathbb{Z}$ as follows:

$$
\ell\left(w \epsilon^{\lambda}\right)=\sum_{\substack{\alpha>0 \\ w(\alpha)<0}}|\langle\alpha, \lambda\rangle+1|+\sum_{\substack{\alpha>0 \\ w(\alpha)>0}}|\langle\alpha, \lambda\rangle| .
$$


This function extends the length function on $W_{a}$. We have a short exact sequence

$$
1 \longrightarrow W_{a} \longrightarrow \tilde{W} \longrightarrow X_{*} / Q^{\vee} \longrightarrow 0
$$

where $Q^{\vee}$ is the coroot lattice, i.e. the subgroup of $X_{*}$ generated by $R^{\vee}$. The restriction of the projection $\widetilde{W} \longrightarrow X_{*} / Q^{\vee}$ to the subgroup $\Omega \subseteq \widetilde{W}$ of elements of length 0 is an isomorphism $\Omega \stackrel{\cong}{\longrightarrow} X_{*} / Q^{\vee}$.

We extend the Bruhat order on $W_{a}$ by declaring

$$
w \tau \leq w^{\prime} \tau^{\prime} \Longleftrightarrow w \leq w^{\prime}, \tau=\tau^{\prime}, \quad w, w^{\prime} \in W_{a}, \tau, \tau^{\prime} \in \Omega .
$$

For an affine root $\beta=\alpha-n, \alpha \in R, n \in \mathbb{Z}$, we have the hyperplane $H_{\beta}=H_{\alpha, n}=$ $\left\{x \in X_{*, \mathbb{R}} ;\langle\alpha, x\rangle=n\right\}$ in $X_{*, \mathbb{R}}:=X_{*} \otimes_{\mathbb{Z}} \mathbb{R}$. An alcove is a connected component of the complement of the union of all affine root hyperplanes inside $X_{*, \mathbb{R}}$. The choice of $\Delta$ determines a base alcove a which is the unique alcove contained in the dominant finite Weyl chamber whose closure contains the origin. The group $\widetilde{W}$ acts on $X_{*, \mathbb{R}}$, and since the union of all affine root hyperplanes is stable under this action, we have an action of $\widetilde{W}$ on the set of alcoves. The affine Weyl group $W_{a}$ acts simply transitively on the set of alcoves, so we can identify $W_{a}$ with the set of alcoves in the standard apartment $X_{*, \mathbb{R}}$ by mapping $w \in W_{a}$ to the alcove $w \mathbf{a}$. On the other hand, the group $\Omega$ of elements of length 0 in $\widetilde{W}$ is precisely the stabilizer of the base alcove inside $\widetilde{W}$. If $\lambda$ denotes the image of the origin under $\tau \in \Omega$, then $\tau w_{0} w=\varepsilon^{\lambda}$, where $w$ is the longest element in the stabilizer $W_{\lambda}$ of $\lambda$ in $W$, and $w_{0}$ is the longest element in $W$.

Define an equivalence relation on $X_{*, \mathbb{R}}$ by saying that $x \sim y$ if and only if for each affine root hyperplane $H$, either $x, y \in H$, or $x$ and $y$ lie in the same half-space of $X_{*, \mathbb{R}}$ with respect to $H$. The equivalence classes are called facets. The support of a facet is the affine subspace it generates. The alcoves are precisely the facets whose support is $X_{*, \mathbb{R}}$, and the facets whose support has codimension 1 are called faces. Given an alcove $\mathbf{a}$, the faces (and facets) contained in the closure of a are called faces (and facets, resp.) of a. The faces of the base alcove correspond bijectively to the simple affine roots, and hence to the elements of $S_{a}$. The concerning element of $S_{a}$ is called the type of the face, and we extend this notion to all faces using the action of $W_{a}$.

Let $r=\# \Delta$ be the semi-simple rank of the root datum. We order the simple reflections in some way and denote them by $s_{1}, \ldots, s_{r}$ in the sequel. The group $\Omega$ acts on the set of simple affine reflections (resp. on the set of simple affine roots) and for $\tau \in \Omega$ we define $\tau(i)$ by $\tau s_{i} \tau^{-1}=s_{\tau(i)}$.

Let us also briefly recall the definition of the affine Hecke algebra. We fix a ground ring $k$, an invertible element $v \in k$ and set $q=v^{2}$. For example, $k$ might be a field (as in [14]), or we could let $k=\mathbb{Z}\left[v, v^{-1}\right]$ be the ring of Laurent polynomials over the integers. We will often write $q^{\frac{1}{2}}$ instead of $v$. The braid group of $\widetilde{W}$ is the group with generators

$$
T_{w}, \quad w \in \widetilde{W}
$$

and relations

$$
T_{w} T_{w^{\prime}}=T_{w w^{\prime}} \text { for } w, w^{\prime} \in \widetilde{W} \text { with } \ell\left(w w^{\prime}\right)=\ell(w)+\ell\left(w^{\prime}\right) .
$$


The affine Hecke algebra $\mathcal{H}$ is the quotient of the group algebra of the braid group (over our fixed ground ring $k$ ), by the two-sided ideal generated by

$$
\left(T_{s}+1\right)\left(T_{s}-q\right), \quad s \in S_{a} .
$$

We denote the image of $T_{w}$ in $\mathcal{H}$ again by $T_{w}$. We sometimes abbreviate $T_{s_{i}}$ to $T_{i}$. Further, it is often useful to use the element $\tilde{T}_{w}:=q^{-\ell(w) / 2} T_{w}$ instead of $T_{w}$. We use $\tilde{T}_{i}$ as an abbreviation for $\tilde{T}_{s_{i}}$.

For $\lambda \in X_{*}$, we define $\Theta_{\lambda} \in \mathcal{H}$ as follows: write $\lambda=\lambda_{1}-\lambda_{2}$ with $\lambda_{1}, \lambda_{2}$ dominant, and let $\Theta_{\lambda}=\tilde{T}_{\epsilon^{\lambda_{1}}} \tilde{T}_{\epsilon^{\lambda_{2}}}^{-1}$ (which is well-defined as an element of $\mathcal{H}$ ).

It is not hard to see that the elements $T_{w}, w \in \widetilde{W}$ form a $k$-basis of $\mathcal{H}$. We note the following lemma which exhibits variants of this basis and which is easily proved by using that $T_{i}^{-1}=q^{-1} T_{i}+\left(q^{-1}-1\right)$.

Lemma 2.1.1 Fix a reduced expression $w=s_{i_{1}} \cdots s_{i_{\ell}} \tau$ for each $w \in \tilde{W}$, and fix signs $\varepsilon_{i}(w) \in\{ \pm 1\}$. Then the set of all elements $T_{i_{1}}^{\varepsilon_{1}(w)} \cdots T_{i_{\ell}}^{\varepsilon_{\ell}(w)} T_{\tau} \in \mathcal{H}$ is a $k$-basis of $\mathcal{H}$.

Similarly, the elements $\Theta_{\lambda} T_{w}, \lambda \in X_{*}, w \in W$ (and likewise the elements $T_{w} \Theta_{\lambda}$ ) form a $k$-basis of $\mathcal{H}$. (See [13], Prop. 3.7, or [9] Lemma 1.7.1.)

2.2 Definition of the alcove walk algebra

The alcove walk algebra $A$ is the (non-commutative) $k$-algebra with generators

$$
c_{i}^{+}, c_{i}^{-}, f_{i}^{+}, f_{i}^{-}, \quad i=0, \ldots, r, \quad t_{\tau}, \tau \in \Omega
$$

and relations

$$
\begin{aligned}
& c_{i}^{-}=c_{i}^{+}+f_{i}^{-}, \quad f_{i}^{-}=-f_{i}^{+}, \quad i=0, \ldots, r, \\
& t_{\tau} ?_{i}=?_{\tau(i)} t_{\tau}, \quad ? \in\left\{c^{+}, c^{-}, f^{+}, f^{-}\right\}, \tau \in \Omega, \\
& t_{\tau} t_{\tau^{\prime}}=t_{\tau \tau^{\prime}}, \quad \tau, \tau^{\prime} \in \Omega
\end{aligned}
$$

The elements $c_{i}^{+}, c_{i}^{-}, f_{i}^{+}, f_{i}^{-}$are called the positive crossing, the negative crossing, the positive folding and the negative folding of type $i$, respectively. We have a natural map $\Phi: A \longrightarrow \mathcal{H}$ from the alcove walk algebra to the affine Hecke algebra by mapping

$$
c_{i}^{+} \mapsto \tilde{T}_{i}, \quad c_{i}^{-} \mapsto \tilde{T}_{i}^{-1}, \quad f_{i}^{+} \mapsto q^{\frac{1}{2}}-q^{-\frac{1}{2}}, \quad f_{i}^{-} \mapsto q^{-\frac{1}{2}}-q^{\frac{1}{2}}, \quad t_{\tau} \mapsto T_{\tau} .
$$

We will determine the kernel of this map (see Proposition 3.2.1), and hence get a new description of the affine Hecke algebra as a certain quotient of the alcove walk algebra.

\subsection{Orientation}

In order to explain the terminology alcove walk algebra, we fix an orientation in the following sense. 
Definition 2.3.1 A root hyperplane orientation is given by distinguishing, for each affine root hyperplane $H$, a positive half-space among the two half-spaces which form the complement of $H$ in $X_{*, \mathbb{R}}$, such that either

(1) for any finite set of affine root hyperplanes, the intersection of the corresponding negative half-spaces is non-empty (and hence contains an alcove), or

(2) for any finite set of affine root hyperplanes, the intersection of the corresponding positive half-spaces is non-empty.

(Here the half-space in $X_{*, \mathbb{R}} \backslash H$ which is not positive, is called negative.)

Given an orientation of type (1), and an alcove $\mathbf{b}$ in the intersection of the negative half-spaces associated to a fixed finite set of affine root hyperplanes, we can say that for these hyperplanes the orientation is given by prescribing that crossing the hyperplane "in the positive direction", i.e. from the negative to the positive half-space, is the same as "walking away" from $\mathbf{b}$.

There are two obvious examples for orientations:

Example 2.3.2 If $\mathbf{b}$ is a fixed alcove, we can use it to define an orientation by saying that for each hyperplane, the negative half-space is the one containing $\mathbf{b}$. We can express this by saying that the most negative point of the orientation lies inside b. Analogously, we get another orientation by saying that for each hyperplane, the positive half-space is the one containing $\mathbf{b}$.

Example 2.3.3 The orientation which will be most important for us is given by calling, for each positive root $\alpha$, and each $j \in \mathbb{Z}$, the half-space

$$
\left\{x \in X_{*, \mathbb{R}} ;\langle x, \alpha\rangle>j\right\}
$$

the positive half-space. In other words (cf. [3]), the negative half-space is the unique half-space which contains a quartier of the form $y+C^{-}$, where $C^{-}$denotes the antidominant Weyl chamber. We can describe this orientation by saying that the most negative point lies infinitely deep in the anti-dominant chamber. We will call this orientation the standard orientation; it is the one used in [14]. It also occurs in the paper [8] by Gaussent and Littelmann.

Alternatively, we could replace the anti-dominant chamber by any other Weyl chamber. Orientations of this type implicitly play a role in [7].

\subsection{Alcove walks}

We will now give a formal definition of the notion of alcove walk, and simultaneously define the end-point end $(\gamma) \in \widetilde{W}$ for an alcove walk $\gamma$. For a more "pictorial", and probably more accessible definition, see [14].

Definition 2.4.1 Fix an orientation as defined in the previous section. Alcove walks are pairs $(w, \tau)$ where $w$ is a word in the $c_{i}^{+}, c_{i}^{-}, f_{i}^{+}, f_{i}^{-}, i \in\{0, \ldots, r\}$, and where $\tau \in \Omega$, subject to certain conditions. The length of the alcove walk is by definition the length of the word $w$. We define the conditions an alcove walk has to satisfy by induction on its length. 
(1) If $\tau \in \Omega$, then $(\emptyset, \tau)$ is an alcove walk of length 0 (where $\emptyset$ denotes the empty word). Its end point is end $((\emptyset, \tau))=\tau \in \widetilde{W}$.

(2) If $(w, \tau)$ is an alcove walk, with $w=w_{1} \cdots w_{n}$ a word of length $n$, and $w_{n+1} \in$ $\left\{c_{i}^{+}, c_{i}^{-}, f_{i}^{+}, f_{i}^{-}\right\}$, such that either

- end $((w, 0)) \mathbf{a}$ is on the negative side of the wall of type $i$ adjacent to the alcove end $((w, 0)) \mathbf{a}$, and $w_{n+1} \in\left\{c_{i}^{+}, f_{i}^{-}\right\}$, or

- end $((w, 0)) \mathbf{a}$ is on the positive side of the wall of type $i$ adjacent to the alcove end $((w, 0)) \mathbf{a}$, and $w_{n+1} \in\left\{c_{i}^{-}, f_{i}^{+}\right\}$,

then $\left(w w_{n+1}, \tau\right)$ is an alcove walk, and its end point is

$$
\operatorname{end}\left(\left(w w_{n+1}, \tau\right)\right)= \begin{cases}\operatorname{end}((w, 0)) s_{i} \tau & \text { if } w_{n+1} \in\left\{c_{i}^{+}, c_{i}^{-}\right\} \\ \operatorname{end}((w, \tau)) & \text { if } w_{n+1} \text { has the form } f_{i}^{ \pm}\end{cases}
$$

(3) All alcove walks arise in this way.

Since we put the $\Omega$-part into the second component, the property of being an alcove walk is actually independent of the $\Omega$-component, and furthermore for the end points we have end $((w, \tau))=\operatorname{end}((w, 0)) \tau$. Because in the definition given here, we build the walks "from left to right", we always have to insert the next step between the given walk and the $\tau$, so that the relevant information about the orientation of the adjacent walls is given by end $((w, 0))$ rather than by end $((w, \tau))$.

The sequence of end points

$$
\operatorname{end}\left(\left(w_{1}, 0\right)\right), \operatorname{end}\left(\left(w_{1} w_{2}, 0\right)\right), \ldots, \operatorname{end}\left(\left(w_{1} \ldots w_{n}, 0\right)\right) \in W_{a}
$$

should be seen as a sequence of alcoves in the standard apartment $X_{*, \mathbb{R}}$-hence the name alcove walk.

We call an alcove walk $(w, \tau)$ non-folded, if no symbols of the form $f_{i}^{+}, f_{i}^{-}$occur in the word $w$. We consider alcove walks as elements of the alcove walk algebra in the obvious way. As the following lemma shows, we can see the choice of orientation as the choice of a basis of the alcove walk algebra.

Lemma 2.4.2 The set of alcove walks is a basis of the alcove walk algebra as a $k$-module.

Proof This is [14], Lemma 3.1; since Ram does not give a proof, for the convenience of the reader we produce a proof here. To simplify the notation, let us suppose that $\Omega=\{0\}$. Because of the relations $c_{i}^{-}=c_{i}^{+}+f_{i}^{-}, f_{i}^{+}=-f_{i}^{-}$, it is clear that $A$ is isomorphic to the free algebra with generators $c_{i}^{+}, f_{i}^{-}$. Hence the set $\mathcal{B}$ of words in $\left\{c_{i}^{+}, f_{i}^{-} ; i=0, \ldots, r\right\}$ is a $k$-basis of $A$. Now fix an orientation and denote by $W$ the set of alcove walks. We have an obvious bijection $\mathcal{W} \longrightarrow \mathscr{B}$ by mapping each walk to the element of $\mathcal{B}$ obtained by changing all exponents of $c$ 's to + , and all exponents of $f$ 's to - . We order $\mathcal{W}$ in some way, such that whenever a walk $w_{1}$ has more $c$ 's in it than a walk $w_{2}$, then $w_{1}>w_{2}$. The bijection $\mathfrak{W} \cong \mathscr{B}$ induces an order on $\mathscr{B}$ with the same property. Now if we express each walk in $\mathcal{W}$ in terms of the basis $\mathscr{B}$ and take the coefficients as the column vector of an (infinite) square matrix (using the order we defined), then this matrix will be upper triangular with 1's on the diagonal, and it follows that $\mathcal{W}$ is a basis as well. 


\section{The independence result}

\subsection{Product expressions}

We again fix an orientation as defined in Sect. 2.3. Given a word $s_{i_{1}} \cdots s_{i_{k}} \tau$ (not necessarily reduced) in the extended affine Weyl group, we can associate to it, or to the corresponding gallery, a unique non-folded alcove walk $c_{i_{1}}^{\varepsilon_{1}} \cdots c_{i_{k}}^{\varepsilon_{k}} t_{\tau}, \varepsilon_{i} \in\{+,-\}$. On the other hand, we can associate to the given word the element

$$
T_{i_{1}}^{\varepsilon_{1}} \cdots T_{i_{k}}^{\varepsilon_{k}} T_{\tau}
$$

in the affine Hecke algebra. We also denote this element by

$$
T_{i_{1}}^{\varepsilon} \cdots T_{i_{k}}^{\varepsilon} \tau
$$

i.e. we let $\varepsilon$ denote the appropriate sign, depending on $v \in\{1, \ldots, k\}$.

As a variant, we can consider alcove walks which do not start at the base alcove, but at another alcove, say at $w \mathbf{a}$. We will denote by

$$
T_{i_{1}}^{\varepsilon(w)} \cdots T_{i_{k}}^{\varepsilon(w)}
$$

the corresponding element in $\mathcal{H}$, where again $\varepsilon(w)$ is understood to vary with $v \in$ $\{1, \ldots, k\}$.

Theorem 3.1.1 Let $w \in \widetilde{W}$ be an element in the extended affine Weyl group, and let

$$
w=s_{i_{1}} \cdots s_{i_{k}} \tau=s_{j_{1}} \cdots s_{j_{\ell}} \tau
$$

be expressions for $w$ (which need not be reduced). Then in the affine Hecke algebra, we have the equality

$$
T_{i_{1}}^{\varepsilon} \cdots T_{i_{k}}^{\varepsilon} T_{\tau}=T_{j_{1}}^{\varepsilon} \cdots T_{j_{\ell}}^{\varepsilon} T_{\tau}
$$

Proof We clearly may assume that $\tau=$ id, i.e. that $w$ actually is an element of the affine Weyl group $W_{a}$. The affine Weyl group is a Coxeter group, and its presentation by generators and relations implies that we can get the expression $s_{j_{1}} \cdots s_{j_{\ell}}$ from $s_{i_{1}} \cdots s_{i_{k}}$ by applying transformations of the following kinds (in a suitable order):

(1) nil-move: delete a subexpression of the form $s_{i} s_{i}$ from the word

(2) inverse nil-move: insert a subexpression of the form $s_{i} s_{i}$ somewhere

(3) braid move: replace a subexpression $s_{i} s_{i^{\prime}} s_{i} \cdots$ by $s_{i^{\prime}} s_{i} s_{i^{\prime}} \cdots$, where both these words consist of $m_{i, i^{\prime}}$ letters, $m_{i, i^{\prime}}$ being the entry in the Coxeter matrix corresponding to $i, i^{\prime}$.

(In fact a much stronger property, the so-called word property holds: we can get from one reduced expression to any other reduced expression using only braid moves (see [17], [1], Theorem 3.3.1).)

Hence it is enough to prove that these types of transformations do not change the product $T_{i_{1}}^{\varepsilon} \cdots T_{i_{k}}^{\varepsilon}$ (of course the signs ${ }^{\varepsilon}$ have to be taken into account). For nil-moves 
and inverse nil-moves this is obvious, since the two adjacent $T$ 's will have exponents 1 and -1 (or -1 and 1 ), thus will cancel.

What remains to show is that for all $i, j$, and for all $w \in W_{a}$, we have

$$
T_{i}^{\varepsilon(w)} T_{j}^{\varepsilon(w)} T_{i}^{\varepsilon(w)} \cdots=T_{j}^{\varepsilon(w)} T_{i}^{\varepsilon(w)} T_{j}^{\varepsilon(w)} \cdots,
$$

where both products have $m_{i, j}$ factors. (Of course, in the case that all the $\varepsilon(w)$ 's are equal, the equality follows immediately from the braid relations in the affine Weyl group.)

Since only finitely many alcoves are involved in the alcove walk, by the definition of orientation, there is an alcove $\mathbf{b}=v \mathbf{a}, v \in W_{a}$, such that the positive/negative direction is determined by whether we are approaching $\mathbf{b}$, or not. We may assume without loss of generality that $\mathbf{b}$ is the most negative point (rather than the most positive point) for the finitely many hyperplanes involved, because otherwise we could replace the orientation by its "inverse": the equality we have to check is the same for both of these orientations. Denote by o the orientation given by making the base alcove the most negative point, and by $\varepsilon_{\mathbf{o}}$ the signs defined with respect to $\mathbf{o}$. We then have

$$
\varepsilon(w)=\varepsilon_{\mathbf{0}}\left(v^{-1} w\right)
$$

in the obvious sense. Hence it is enough to check the assertion of the theorem for the orientation $\mathbf{o}$, i.e. we may assume that the signs are determined by whether we come closer to a fixed point in the interior of the base alcove, or not; in other words whether the length of the element in the affine Weyl group corresponding to the alcove decreases, or increases.

The coset $w W_{i, j}$ of the parabolic subgroup $W_{i, j} \subseteq W_{a}$ generated by $s_{i}, s_{j}$ has a unique element of minimal length, and a unique element of maximal length, and there are two ways to go from the minimal length element to the maximal length element. We multiply (3.1.1) on the right by the inverse of the right hand side, and get an equation of the form

$$
T_{i}^{\varepsilon_{1}} T_{j}^{\varepsilon_{2}} \cdots T_{j}^{\varepsilon_{2 m_{i, j}}}=1,
$$

and the alcove walk corresponding to the left hand side of this equation starts at $w \mathbf{a}$ and then comes back to $w \mathbf{a}$, seeing each alcove in the coset $w W_{i, j}$ exactly once. Since the situation is symmetric, we may and will assume that we start in the positive direction. Then we continue in the positive direction until we get to the element of maximal length, from there we go in the negative direction until we get to the element of minimal length, and finally go in the positive direction back to wa, where we started.

The string of $T$ 's corresponding to the part of the walk going from the maximal to the minimal element is a string of length $m_{i, j}$, and all $T$ 's have an exponent -1 . We may hence replace this string with the string which has $i$ and $j$ exchanged because of the braid relations in the affine Weyl group, and we then get that the whole product cancels.

Note that the statement of the proposition remains true, if we replace $T_{i}$ by $\tilde{T}_{i}$ everywhere (the same proof applies). 


\subsection{The kernel of $\Phi$}

As before, we fix an orientation. Recall that the notion of alcove walk depends on the orientation, but that neither the definition of the alcove walk algebra, nor the morphism $\Phi: A \longrightarrow \mathcal{H}$ do. Therefore the following proposition is a little surprising.

Proposition 3.2.1 The kernel of $\Phi: A \longrightarrow \mathcal{H}$ is the two-sided ideal $g \subseteq$ A generated by

$$
\begin{aligned}
& f_{i}^{+}-\left(q^{\frac{1}{2}}-q^{-\frac{1}{2}}\right), \quad i=0, \ldots, r \\
& p-p^{\prime} \text { for } p, p^{\prime} \text { non-folded alcove walks with the same end point. }
\end{aligned}
$$

We remark that this definition implies in particular that $c_{i}^{-}-\left(c_{i}^{+}\right)^{-1} \in \mathcal{g}$ for all $i$ (of course, these elements obviously lie in $\operatorname{ker} \Phi$ ).

Proof Theorem 3.1.1 shows that $\Phi$ factors through a morphism $A / \mathcal{G} \longrightarrow \mathcal{H}$ which of course is again surjective. Fixing a non-folded walk $p_{w}$ from a to $w \mathbf{a}$ for each $w \in \widetilde{W}$ gives us a set of elements in $A / \mathcal{g}$ which generates $A / \mathcal{g}$ as a $k$-module. Lemma 2.1.1 implies that this set is mapped to a basis of $\mathcal{H}$, and hence the morphism $A / \mathcal{g} \longrightarrow \mathcal{H}$ is an isomorphism.

Each choice of orientation hence gives us a basis of $\mathcal{H}$ consisting of the images of non-folded walks to $w \mathbf{a}, w \in \widetilde{W}$, so in a sense the choice of orientation corresponds to the choice of a basis for $\mathcal{H}$; cf. Remark 3.6 in [14]. Of course we do not get every basis of $\mathcal{H}$ in this way.

It is easy to see (and not surprising) that neither Theorem 3.1.1 nor the proposition above hold for "orientations" which do not satisfy the condition imposed in Sect. 2.3.

Remark 3.2.2 The notion of alcove walk (for the standard orientation) is related to the Bruhat-Tits building. Assume that $q$ is the number of elements of the residue class field of a local field $K$, and fix a split reductive algebraic group $G$ over $K$ which gives rise to the root system under consideration.

Denote by $\rho$ the retraction of the Bruhat-Tits building to the standard apartment from an alcove "far out" in the anti-dominant chamber. More precisely, for each alcove in the building, its image under such retractions will depend on the alcove, but will stabilize if the alcove is sufficiently deep in the anti-dominant chamber, and this gives us the image of the alcove under $\rho$. See [3] 2.9.1.

Then to each non-stuttering gallery in the building (starting at the base alcove) we can associate via this retraction a unique alcove walk. Let $\mathbf{b}$ be an alcove in the building which is part of such a gallery. If $\rho(\mathbf{b})$ is on the positive side of the wall of type $i$ adjacent to it, then there are $q-1$ alcoves $\mathbf{b}^{\prime}$ adjacent to $\mathbf{b}$, but different from it, such that $\rho\left(\mathbf{b}^{\prime}\right)=\rho(\mathbf{b})$, and the alcove walk will have a positive folding precisely if one of these alcoves $\mathbf{b}^{\prime}$ is the successor of $\mathbf{b}$ in the gallery. This point of view is explained in more detail in [8], see also [15]. 


\subsection{The Bernstein relations}

In this section we work with the standard orientation, i.e. we put the most negative point infinitely deep in the anti-dominant chamber. We define elements $t_{w}, \theta_{\lambda} \in \mathcal{H}$ ( $w \in W, \lambda \in X_{*}$ ) as follows. For $w \in W$, let $p \in A$ be a non-folded alcove walk from a to $w^{-1} \mathbf{a}$, and let $t_{w}:=\Phi(p)^{-1} \in \mathcal{H}$.

For $\lambda \in X_{*}$, denote by $\theta_{\lambda} \in \mathcal{H}$ the image under $\Phi$ of a non-folded alcove walk with end point $\epsilon^{\lambda}$.

The following proposition is Proposition 3.2 in [14]. It shows that the Bernstein relations are satisfied for the elements $\theta_{\lambda}, t_{w}$ in $\widetilde{H}$. See also [16], Example 6.3, where some special cases are considered.

Proposition 3.3.1 Let $\tau \in \Omega, \lambda, \mu \in X_{*}, w \in W$, and $1 \leq i \leq r$, and denote by $\alpha_{i}$ the corresponding simple root. Let $\tilde{\alpha} \in R$ be the positive root such that $H_{\alpha_{0}}=H_{\tilde{\alpha}, 1}:=$ $\left\{x \in X_{*, \mathbb{R}} ;\langle\tilde{\alpha}, x\rangle=1\right\}$ is the wall of $\mathbf{a}$ which is not a wall of the Weyl chamber $\mathbf{a}$ lies in. Then we have

(1) $\theta_{\lambda} \theta_{\mu}=\theta_{\lambda+\mu}$

(2) $t_{s_{i}} t_{w}= \begin{cases}t_{s_{i} w} & \text { if } \ell\left(s_{i} w\right)>\ell(w) \\ t_{s_{i} w}+\left(q^{\frac{1}{2}}-q^{-\frac{1}{2}}\right) t_{w} & \text { otherwise }\end{cases}$

(3) $t_{s_{i}} \theta_{\lambda}=\theta_{s_{i} \lambda} t_{s_{i}}+\left(q^{\frac{1}{2}}-q^{-\frac{1}{2}}\right) \frac{\theta_{\lambda}-\theta_{s_{i} \lambda}}{1-\theta_{-\alpha_{i}}}$

(4) $\Phi\left(c_{0}^{+}\right) t_{s_{\tilde{\alpha}}}=\theta_{\tilde{\alpha}^{\vee}}$, where $s_{\tilde{\alpha}}$ denotes the reflection associated with $\tilde{\alpha}$.

(5) Let $\tau \in \Omega$ be an element of $\widetilde{W}$ of length 0 . Recall that $\tau$, as an automorphism of $X_{*, \mathbb{R}}$ maps the base alcove to itself. Denote by $\lambda \in X_{*}$ the image of the origin under $\tau$. Then

$$
\theta_{\lambda}=T_{\tau} t_{w_{0} w},
$$

where $w$ is the longest element in the stabilizer $W_{\lambda}$ of $\lambda$ in $W$, and $w_{0}$ is the longest element in $W$.

Proof All these relations can be checked with relatively little effort in terms of alcove walks. We give some of the details, since the proof in [14] is partly quite terse. If $p_{\lambda}$ is a non-folded walk from $\mathbf{a}$ to $\epsilon^{\lambda} \mathbf{a}$, and $p_{\mu}$ is a non-folded walk from $\mathbf{a}$ to $\epsilon^{\mu} \mathbf{a}$, then clearly the composition $p_{\lambda} p_{\mu}$ is a non-folded walk from a to $\epsilon^{\lambda+\mu} \mathbf{a}$, and this gives (1).

To get (3), we may assume without loss of generality that $\left\langle\alpha_{i}, \lambda\right\rangle \geq 0$. Let $p_{\lambda}=$ $\left(c_{i_{1}}^{\varepsilon_{1}} \cdots c_{i_{\ell}}^{\varepsilon_{\ell}}, \tau\right)$ be a walk from a to $\epsilon^{\lambda} \mathbf{a}$ of minimal length. Let $\mathbf{a}_{1}=\mathbf{a}, \mathbf{a}_{2}=s_{i_{1}} \mathbf{a}$, $\ldots, \mathbf{a} \ell=s_{i_{1}} \cdots s_{i_{\ell}} \mathbf{a}$ be the sequence of alcoves "visited" by this path. Consider the element $c_{i}^{-} p_{\lambda} \in \mathcal{H}$; the corresponding sequence of alcoves is the base alcove plus the mirror image of the sequence $\mathbf{a}_{1}, \ldots, \mathbf{a}_{\ell}$ with respect to the reflection $s_{i}$. It is not an alcove walk in general, since some of the $c_{i_{v}}$ will now carry the wrong exponents; the places where this will happen are precisely those where the wall between $\mathbf{a}_{v-1}$ and $\mathbf{a}_{v}$ has the form $H_{\alpha_{i}, k}$ for some $k \in \mathbb{Z}$ - we call those $v$ relevant. Since $\left\langle\alpha_{i}, \lambda\right\rangle \geq 0$, all of those walls are crossed in the positive direction, i.e. $\varepsilon_{v}=1$ for all relevant $v$. We want to write $c_{i}^{-} p_{\lambda}$ as a sum of walks. Let $v$ be the minimal relevant index. Since $c_{i_{v}}^{+}=c_{i_{v}}^{-}+f_{i_{v}}^{+}$, we have $c_{i}^{-} p_{\lambda}=p_{1}+q_{1}$, where $p_{1}$ is obtained by replacing 
$c_{i_{v}}^{+}$in $c_{i}^{-} p_{\lambda}$ by $c_{i_{v}}^{-}$, and $q_{1}$ is obtained by replacing it by $f_{i_{v}}^{+}$. Then $q_{1}$ is indeed an alcove walk, and its end point is $s_{\alpha_{i}+1} s_{\alpha_{i}} \epsilon^{\lambda} \mathbf{a}=\epsilon^{\lambda-\alpha_{i}^{\vee}} \mathbf{a}$, and since there is precisely one (positive) folding in $q_{1}$, in $\mathcal{H}$ the element $q_{1}$ is equal to $\left(q^{\frac{1}{2}}-q^{-\frac{1}{2}}\right) \theta_{\lambda-\alpha_{i}^{\vee}}$. On the other hand, $p_{1}$ will not be an alcove walk, in general, and we have to repeat this procedure for the next relevant index. Since $t_{i}=\Phi\left(c_{i}^{-}\right)^{-1}$, we have proved that

$$
t_{i}^{-1} \theta_{\lambda}=c_{i}^{-} p_{\lambda}=\left(q^{\frac{1}{2}}-q^{-\frac{1}{2}}\right) \theta_{\lambda-\alpha_{i}^{\vee}}+\cdots+\left(q^{\frac{1}{2}}-q^{-\frac{1}{2}}\right) \theta_{\lambda-\left\langle\alpha_{i}, \lambda\right\rangle \alpha_{i}^{\vee}}+\theta_{s_{i} \lambda} t_{i}^{-1},
$$

and since $t_{i}^{-1}=t_{i}+q^{-\frac{1}{2}}-q^{\frac{1}{2}}$, we get

$$
t_{i} \theta_{\lambda}=\left(q^{\frac{1}{2}}-q^{-\frac{1}{2}}\right)\left(\theta_{\lambda}+\theta_{\lambda-\alpha_{i}^{\vee}}+\cdots+\theta_{\lambda-\left(\left\langle\alpha_{i}, \lambda\right\rangle-1\right) \alpha_{i}^{\vee}}\right)+\theta_{s_{i} \lambda} t_{i},
$$

which is the identity we had to prove.

From the definitions it is clear that $t_{s_{i}}=\Phi\left(c_{i}^{-}\right)^{-1}=\tilde{T}_{i}$ for $i=1, \ldots, r$. It is also clear that $\Theta_{\lambda}=\theta_{\lambda}$ for $\lambda$ dominant or anti-dominant, and part (1) of the proposition implies that $\Theta_{\lambda}=\theta_{\lambda}$ holds in general. Finally, it follows from part (4) that $\Phi\left(c_{0}^{+}\right)=$ $\tilde{T}_{0}$. Since the elements $\Theta_{\lambda} T_{w}, \lambda \in X_{*}, w \in W$ form a basis of $\mathcal{H}$, we have obtained a proof of the Bernstein presentation of $\mathcal{H}$.

Remark 3.3.2 The way of reasoning in [14] is different from the above. Ram's Proposition [14] 3.2 (= Prop. 3.3.1 above) shows (taking the Bernstein presentation of the affine Hecke algebra as a piece of input), that the surjections from the alcove walk algebra induce a (surjective) morphism $\mathcal{H} \longrightarrow \widetilde{H}$.

The injectivity follows from Schwer's results ([16], Lemma 6.1; see also [15]) or from Theorem 3.1.1 above. Another way to approach this question is the following: It is clear that the quotient of $A$ by the free $k$-submodule generated by the elements $f_{i}^{+}-\left(q^{\frac{1}{2}}-q^{-\frac{1}{2}}\right), i=0, \ldots, r$ and $p-p^{\prime}$ for $p, p^{\prime}$ non-folded alcove walks with $\operatorname{end}(p)=\operatorname{end}\left(p^{\prime}\right)$, admits a basis of the form $\theta_{\lambda} t_{w}, \lambda \in X_{*}, w \in W$; hence it would be enough to show that this $k$-submodule actually is an ideal. (Again, it is clear from hindsight that this is true.)

\subsection{The case of unequal parameters}

To keep the notation simple, we have considered only the case of equal parameters. However, we can in a similar way as above consider a variant of the alcove walk algebra with a parameter system. More precisely, assume that a parameter system $L: \widetilde{W} \longrightarrow \mathbb{Z}_{\geq 0}$ is given; we use the notation of Lusztig [13]. Denote by $\mathcal{H}$ the Hecke algebra associated with this system of parameters; see loc. cit. It is clear from the proof that Theorem 3.1.1 holds in this case, too, when we use the elements $T_{i}$, and it is easy to see that as before it holds with the $\tilde{T}_{i}=q^{-\frac{L\left(s_{i}\right)}{2}} T_{i}$, as well. The alcove walk algebra $A$ does not change, but we change the map $\Phi: A \longrightarrow \mathcal{H}$, namely we map

$$
\begin{aligned}
& c_{i}^{+} \mapsto \tilde{T}_{i}, \quad c_{i}^{-} \mapsto \tilde{T}_{i}^{-1}, \\
& f_{i}^{+} \mapsto q^{\frac{L\left(s_{i}\right)}{2}}-q^{-\frac{L\left(s_{i}\right)}{2}}, \quad f_{i}^{-} \mapsto q^{-\frac{L\left(s_{i}\right)}{2}}-q^{\frac{L\left(s_{i}\right)}{2}}, \quad t_{\tau} \mapsto T_{\tau} .
\end{aligned}
$$


As above, $\Phi$ induces an isomorphism $A / \mathcal{g} \cong \mathcal{H}$, where $\mathcal{g} \subseteq A$ is the two-sided ideal generated by the elements $f_{i}^{+}-\left(q^{\frac{L\left(s_{i}\right)}{2}}-q^{-\frac{L\left(s_{i}\right)}{2}}\right), i=0, \ldots, r$, and $p-p^{\prime}$ where $p$, $p^{\prime}$ are non-folded alcove walks with the same end point.

It is slightly more difficult to prove the Bernstein relations in $A / g$ as in Proposition 3.3.1 in this more general case in terms of alcove walks. We have to prove that

$$
t_{s_{i}} \theta_{\lambda}= \begin{cases}\theta_{s_{i} \lambda} t_{s_{i}}+\left(q^{\frac{L\left(s_{i}\right)}{2}}-q^{-\frac{L\left(s_{i}\right)}{2}}\right) \frac{\theta_{\lambda}-\theta_{s_{i} \lambda}}{1-\theta_{-\alpha_{i}^{\vee}}} & \text { if } \alpha \notin 2 X^{*}, \\ \left(\left(q^{\frac{L\left(s_{i}\right)}{2}}-q^{-\frac{L\left(s_{i}\right)}{2}}\right)+\theta_{-\alpha_{i}^{\vee}}\left(q^{\frac{L\left(\tilde{s}_{i}\right)}{2}}-q^{-\frac{L\left(\tilde{s}_{i}\right)}{2}}\right)\right) \frac{\theta_{\lambda}-\theta_{s_{i} \lambda}}{1-\theta_{-2 \alpha_{i}^{\vee}}} & \text { if } \alpha \in 2 X^{*} .\end{cases}
$$

Roughly speaking, the same proof as above applies, but obviously one has to be more careful in order to identify the factors that come from the foldings: we need to know which $i_{v}$ occur for relevant $v$. The following two lemmas provide this information and give a simple explanation for the appearance of the parameter associated to $\tilde{s}$ when $\alpha \in 2 X^{*}$.

Lemma 3.4.1 Let $H \subseteq X_{*, \mathbb{R}}$ be an affine root hyperplane, and let $I \subseteq\{0, \ldots, r\}$ be the set of types of faces with support $H$. Then $\left\{s_{i} ; i \in I\right\}$ is a conjugacy class of simple reflections under the affine Weyl group $W_{a}$.

Proof After applying an element of $W_{a}$ to $H$, if necessary, we may assume that $H$ contains a face of the base alcove, say of type $i_{1}$.

First assume that $F$ is a face with support $H$, of type $i_{2}$, say. Choose $w \in W_{a}$ such that $F$ is a face of $w \mathbf{a}$. Since $F$ is fixed by the reflection with respect to $H$, it is clear that the gallery starting at a and consisting of crossing $H$, crossing the faces of the types described by $w$, and finally crossing $H$ again, leads to $w \mathbf{a}$. In other words, $s_{i_{1}} w s_{i_{2}}=w$, which shows that $s_{i_{1}}$ and $s_{i_{2}}$ are conjugate.

On the other hand, assume that $s_{i_{1}}=w s_{i_{2}} w^{-1}$, i.e. $s_{i_{1}} w=w s_{i_{2}}$. This shows that the image of $w \mathbf{a}$ under $s_{i_{1}}$ (which is just the reflection with respect to $H$ ) is adjacent to $w \mathbf{a}$ by a face of type $i_{2}$. Since the alcoves are adjacent, and $H$ lies between them, this face has to lie on $H$.

The lemma shows that we can associate to each affine root hyperplane a parameter $L(H):=L\left(s_{i}\right)$, where $i$ is the type of any face with support $H$.

Lemma 3.4.2 Now suppose that $\alpha_{i}$ is a simple root.

(1) If $\alpha_{i} \notin 2 X^{*}$, then for all $j \in \mathbb{Z}$, we have $L\left(H_{\alpha_{i}, j}\right)=L\left(s_{i}\right)$.

(1) If $\alpha_{i} \in 2 X^{*}$, then for $j$ even, $L\left(H_{\alpha_{i}, j}\right)=L\left(s_{i}\right)$, and for $j$ odd, $L\left(H_{\alpha_{i}, j}\right)=L\left(\tilde{s}_{i}\right)$ (where we again use the notation of [13]).

Proof Consider the map $\mathbb{Z} \longrightarrow \mathbb{Z}_{\geq 0}$ mapping $j$ to $L\left(H_{\alpha_{i}, j}\right)$. Since the types of faces are preserved by the action of $W_{a}$, and since $s_{\alpha_{i}, j}\left(H_{\alpha_{i}, j-1}\right)=H_{\alpha_{i}, j+1}$, the map factors through $\mathbb{Z} / 2 \mathbb{Z}$. Furthermore, the conjugacy class corresponding to $H_{\alpha_{i}, 0}$ is clearly the conjugacy class of $s_{i}$, so 0 maps to $L\left(s_{i}\right)$. 
Now if $\alpha_{i} \notin 2 X^{*}$, then there exists $\lambda \in X_{*}$ with $\left\langle\alpha_{i}, \lambda\right\rangle$ odd. Consider the translation of $H_{\alpha_{i}, 0}$ by $\lambda$. We have

$$
L\left(H_{\alpha_{i}, 0}\right)=L\left(\epsilon^{\lambda} H_{\alpha_{i}, 0}\right)=L\left(H_{\alpha_{i}, 1}\right) .
$$

If $\lambda$ is a coroot, such that translation by $\lambda$ is an element of the affine Weyl group, then the previous lemma shows that the conjugacy classes under $W_{a}$ associated with $H_{\alpha_{i}, 0}$ and $\epsilon^{\lambda} H_{\alpha_{i}, 0}$ coincide. In general, $\epsilon^{\lambda} \in \widetilde{W}$, and the first equality follows from the fact that simple reflections conjugate under $\widetilde{W}$ have the same parameter. The second equality is clear since the pairing of $\alpha_{i}$ and $\lambda$ is odd.

Finally, we consider the case $\alpha_{i} \in 2 X^{*}$. This means that we are in a very special case (cf. [13], 2.4). The Coxeter system $\left(W_{a}, S_{a}\right)$ is of type $\tilde{C}_{r}$, we may assume the root datum is simply connected, and $\tilde{s}_{i}$ is the affine simple reflection $s_{0}$ (i.e. $\alpha_{i}=\alpha_{r}$ with the notation of [2]). Denoting by $\tilde{\alpha}$ the highest root, we have $\alpha_{i}+\tilde{\alpha}=2 \beta$ for a $\operatorname{root} \beta$, and the image of $H_{\alpha_{i},-1}$ under the reflection $s_{\beta}$ is $H_{\tilde{\alpha}, 1}$. Clearly, the conjugacy class associated with $H_{\tilde{\alpha}, 1}$ is the conjugacy class of $s_{0}$, so the lemma is proved.

\section{Applications to nearby cycles sheaves on affine flag manifolds}

As is well known, the combinatorics of affine Hecke algebras is intimately connected with algebraic geometric questions. The alternating trace of Frobenius on the stalks of Iwahori-equivariant (perverse) sheaves on the affine flag manifold can be considered as an element of the Iwahori-Hecke algebra - this is an instance of Grothendieck's sheaf-function dictionary. In this section we point out an application of the theory of alcove walks to a geometric question, and state some further combinatorial questions on the affine Hecke algebra related to affine flag varieties and the reduction of Shimura varieties.

\subsection{Existence of minimal expressions}

We say that $\Theta_{\lambda} \in \mathcal{H}$ has a minimal expression, if we can express it in the form

$$
\Theta_{\lambda}=\tilde{T}_{i_{1}}^{\varepsilon_{1}} \cdots \tilde{T}_{i_{\ell}}^{\varepsilon_{\ell}} \tilde{T}_{\tau}
$$

where $\varepsilon_{i} \in\{ \pm 1\}$ and where $s_{i_{1}} \cdots s_{i_{\ell}} \tau$ is a reduced expression in $\widetilde{W}$; cf. the paper [11] by Haines and Pettet, where the case of $\Theta_{\lambda}^{-}$instead of $\Theta_{\lambda}$ is considered, and where minimal expressions are interpreted in a sheaf-theoretic way, using Demazure resolutions of Schubert varieties in the affine flag variety. As an application of Theorem 3.1.1 we get that minimal expressions always exist.

Corollary 4.1.1 Let $\lambda$ be a coweight, and let $\epsilon^{\lambda}=s_{i_{1}} \cdots s_{i_{k}} \tau$ be a reduced expression. Then

$$
\Theta_{\lambda}=\tilde{T}_{i_{1}}^{\varepsilon} \cdots \tilde{T}_{i_{k}}^{\varepsilon} \tilde{T}_{\tau}
$$

Proof By definition

$$
\Theta_{\lambda}=\tilde{T}_{\lambda_{1}} \tilde{T}_{\lambda_{2}}^{-1}
$$


where $\lambda=\lambda_{1}-\lambda_{2}, \lambda_{1}, \lambda_{2}$ dominant, and if we choose reduced expressions $t_{\lambda_{1}}=$ $s_{j_{1}} \cdots s_{j_{\ell}} \tau_{1}, t_{\lambda_{2}}=s_{j_{1}^{\prime}} \cdots s_{j_{\ell^{\prime}}^{\prime}} \tau_{2}$, then it is clear that

$$
\begin{aligned}
\tilde{T}_{\lambda_{1}} \tilde{T}_{\lambda_{2}}^{-1} & =\tilde{T}_{j_{1}} \cdots \tilde{T}_{j_{\ell}} \tilde{T}_{\tau_{1}} \tilde{T}_{\tau_{2}}^{-1} \tilde{T}_{j_{\ell^{\prime}}^{\prime}}^{-1} \cdots \tilde{T}_{j_{1}^{\prime}}^{-1} \\
& =\tilde{T}_{j_{1}}^{\varepsilon} \cdots \tilde{T}_{j_{\ell}}^{\varepsilon} \tilde{T}_{\tau\left(j_{\ell^{\prime}}^{\prime}\right)}^{\varepsilon} \cdots \tilde{T}_{\tau\left(j_{1}^{\prime}\right)}^{\varepsilon} \tilde{T}_{\tau} .
\end{aligned}
$$

Now Theorem 3.1.1 immediately implies the result.

It is clear that in an analogous way we can compute variants of the $\Theta_{\lambda}$, where we write $\lambda$ as a difference not of dominant coweights, but of coweights lying in some other fixed finite Weyl chamber. In particular we see that the elements $\Theta_{\lambda}^{-}$studied in [11] (which are defined by $\Theta_{\lambda}^{-}=\tilde{T}_{\lambda_{1}} \tilde{T}_{\lambda_{2}}^{-1}$, where $\lambda=\lambda_{1}-\lambda_{2}$ with $\lambda_{1}, \lambda_{2}$ antidominant) admit minimal expressions as well.

In the paper [11] by Haines and Pettet, there are several results whose proofs rely on the existence of minimal expressions.

\subsection{Applications}

In [5] the Jordan-Hölder series of certain nearby cycles sheaves which arise naturally in the Beilinson-Gaitsgory deformation for the affine flag manifold to the affine Grassmannian were studied. The trace of Frobenius on the stalks of these sheaves is a polynomial in $q$ and $q^{-1}$, and it encodes information about the singularities occurring in certain closed subschemes of the affine flag variety.

Let us give a definition of these polynomials in terms of the Iwahori-Hecke algebra (for the corresponding group $G$ over a local field). For $\mu \in X_{*}$, we denote by $z_{\mu}$ the Bernstein element $z_{\mu}=\sum_{\lambda \in W \mu} \Theta_{\lambda}$, an element of the center of $\mathcal{H}$. The theorem of Gaitsgory [4], and of Haines-Ngô [10] (in the function field case and the arithmetic case, resp.), which was conjectured by Kottwitz, says that the trace of Frobenius on the nearby cycles sheaf associated with $\mu$ is given by the element

$$
R \Psi_{\mu}=(-1)^{\ell\left(\epsilon^{\mu}\right)} q^{\frac{\ell\left(\epsilon^{\mu}\right)}{2}} \sum_{\lambda \leq \mu} m_{\mu}(\lambda) z_{\lambda},
$$

where $m_{\mu}(\lambda)$ denotes the dimension of the weight space for $\lambda$ of the irreducible representation with highest weight $\mu$ of the dual group of $G$. See for instance [5], 2.7, for further explanations on the geometric background.

Expressed in the context of Hecke algebras, the main focus of [5] is to investigate the expression of $R \Psi_{\mu}$ with respect to the Kazhdan-Lusztig basis; for our purposes the most suitable normalization is to consider the basis

$$
C_{w}^{\prime \prime}=\sum_{x}(-1)^{\ell(x)} P_{x, w} T_{x}, \quad w \in \widetilde{W}
$$

where $P_{x, w}$ denotes the Kazhdan-Lusztig polynomial associated with $x$ and $w$. We define $m(w) \in \mathbb{Z}\left[q, q^{-1}\right]$ by requiring

$$
R \Psi_{\mu}=\sum_{w} m(w) C_{w}^{\prime \prime}
$$


Based on computational evidence, Haines and the author conjectured that the $m(w)$ are actually polynomials, and that $\operatorname{deg} m(w) \leq \ell\left(\epsilon^{\mu}\right)-\ell(w)$. In the case where minimal expressions as above are available, we gave a proof of this conjecture in [5], Sect. 9; the results obtained above show that this proof is valid in general. (Two quite different proofs of this fact are given in [6].)

There are however a number of open questions about these "multiplicity polynomials" $m(w)$. Answers to these questions would improve the understanding of the geometry of certain closed subschemes of the affine flag variety; in some cases this has consequences for the reduction modulo a prime $p$ of certain Shimura varieties.

- It would be desirable to give a "closed formula" for the $m(w)$ of combinatorial nature. So far, except for trivial cases, such a formula is known only in the case $\ell(w)=0$ (which is surprising, since from a geometric point of view this case should be considered as the most complicated one.) A cohomological interpretation for general $w$, which however is not very manageable is given in [5], Cor. 6.2.

- In all cases we computed, if $m(w) \neq 0$, then the constant term of $m(w)$ is 1 , and the bound on the degree given above is sharp. Is this true in general?

- It appears that the polynomial $m(w)$ depends only on the isomorphism type of the Bruhat graph of the set $\{x \in \widetilde{W} ; x \geq w, m(x) \neq 0\}$.

Another type of problem which is not yet sufficiently understood is the dimension of affine Deligne-Lusztig varieties, in the Iwahori case say. This can be seen as a combinatorial problem in the Bruhat-Tits building of the underlying group, and can also be formulated as a problem about the Iwahori-Hecke algebra. See [7].

Acknowledgements I am grateful to Arun Ram for explaining to me his theory of alcove walks, and in particular for showing me the statement of Theorem 3.1.1. I also thank Thomas Haines who pointed out a few inaccuracies in a preliminary version of this text and made several very helpful remarks. Finally, thanks are due to an anonymous referee for a number of helpful remarks, and in particular for pointing me to Schwer's thesis [16].

\section{References}

1. Björner, A., Brenti, F.: Combinatorics of Coxeter Groups, Springer Graduate Texts in Mathematics, vol. 231 (2005)

2. Bourbaki, N.: Groupes et Algèbres de Lie. Chapters IV-VI. Masson, Paris (1981)

3. Bruhat, F., Tits, J.: Groupes réductifs sur un corps local I. Inst. Ht. Études Sci. Publ. Math. 41, 5-251 (1972)

4. Gaitsgory, D.: Construction of central elements in the affine Hecke algebra via nearby cycles. Invent. Math. 144, 253-280 (2001)

5. Görtz, U., Haines, T.: The Jordan-Hölder series for nearby cycles on some Shimura varieties and affine flag varieties, J. Reine Angew. Math. (to appear), math.AG/0402143

6. Görtz, U., Haines, T.: Bounds on weights of nearby cycles and Wakimoto sheaves on affine flag manifolds. Manuscr. Math. 120(4), 347-358 (2006)

7. Görtz, U., Haines, T., Kottwitz, R., Reuman, D.: Dimensions of some affine Deligne-Lusztig varieties. Ann. Sci. de l'E.N.S. 4 Sér. 39, 467-511 (2006)

8. Gaussent, S., Littelmann, P.: LS galleries, the path model, and MV cycles. Duke Math. J. 127(1), 35-88 (2005)

9. Haines, T., Kottwitz, R., Prasad, A.: Iwahori-Hecke algebras, math.RT/0309168

10. Haines, T., Ngô, B.C.: Nearby cycles for local models of some Shimura varieties. Compos. Math. 133, 117-150 (2002) 
11. Haines, T., Pettet, A.: Formulae relating the Bernstein and Iwahori-Matsumoto presentations of an affine Hecke algebra. J. Algebra 252(1), 127-149 (2002)

12. Humphreys, J.: Reflection groups and Coxeter groups, Camb. Stud. Adv. Math. vol. 29. Cambridge University Press, Cambridge (1990)

13. Lusztig, G.: Affine Hecke algebras and their graded version. J. Am. Math. Soc. 2(3), 599-635 (1989)

14. Ram, A.: Alcove walks, Hecke algebras, spherical functions, crystals and column strict tableaux. Pure Appl. Math. Q. 2(4), (2006)

15. Schwer, C.: Galleries, Hall-Littlewood polynomials, and structure constants of the spherical Hecke algebra, Int. Math. Res. Not. 2006, Art. ID 75395 (2006)

16. Schwer, C.: Galleries and $q$-analogs in combinatorial representation theory. PhD Thesis, Univ. zu Köln (2006)

17. Tits, J.: Le problème des mots dans les groupes de Coxeter. In: Symposia Mathematica, INDAM, Rome, 1967/1968, pp. 175-185. Academic Press (1969) 